\title{
Evaluation of Various Methods for Determining Bulk Compositions of Chondrules and Other Objects in Petrographic Thin Sections
}

\author{
Daniel Kent Ross ${ }^{1,2 *}$ and Justin I. Simon ${ }^{2}$ \\ 1. Univ. of Texas at El Paso, El Paso TX., USA and Jacobs Technology, \\ 2. Center for Cosmochemistry and Geochronology, NASA-Johnson Space Center, Houston TX, USA. \\ * Corresponding author: daniel.ross@nasa.gov
}

Studies of many objects in petrographic thin section, such as melt inclusions in igneous rocks, chondrules and $\mathrm{Ca}-\mathrm{Al}$ rich inclusions in chondritic meteorites, or clasts in lunar and other breccias, require or can benefit from knowledge of their bulk compositions. Given the scarcity of these materials, the reluctance of curators to provide more abundant material, and the extreme difficulty of cleanly separating such objects from their rock matrices, geochemical and cosmochemical studies need the ability to determine their bulk compositions from in situ methods, such as defocused beam analysis, or quantitative chemical mapping by electron beam methods.

The traditional method of obtaining bulk compositions has been by defocused beam analysis of many spots on the object of interest, with averaging of these spots to produce the final composition. This method has several potential drawbacks. The assumption built into matrix correction procedures, such as ZAF [1], PAP [2] and PRZ [3] is that all the X-ray signal derived from exposure to the beam is produced from a region of uniform composition. This assumption is clearly violated in the defocused beam method. The objects are usually multiphase, such that the beam often excites x-ray emission from more than one phase at a time. Thus, the fundamental assumption of the matrix correction scheme is violated. Moreover, the defocused beam method limits one's ability to collect data near the margins of the object, because of the need to prevent spilling the beam over into regions outside the object of interest.

In this study, the results of three methods used to determine bulk compositions are compared, and the drawbacks and advantages of each method are discussed. These methods have been applied to several Al-rich chondrules (ARCs) from the ordinary chondrite NWA7402. Data are presented from the WDS-defocused beam method, also from x-ray mapping by ED-SDD (energy dispersive-silicon drift detector), and finally from xray mapping with WDS detection. All these data were obtained using the JEOL $8530 \mathrm{~F}$ electron probe at Johnson Space Center-NASA. Defocused beam-WDS data $(10 \mu \mathrm{m}$ beam $)$ were obtained by averaging $>100$ spots and reduced using the JEOL ZAF routine, with beam energy of $15 \mathrm{kV}$, and $30 \mathrm{nA}$ beam current. EDSDD data were collected and processed using the ThermoFisher NSS software, with PRZ matrix corrections, using $15 \mathrm{kV}$ energy and $40 \mathrm{nA}$ current. The objects of interest were digitized within the Thermo-Fisher software, and all x-ray counts from the digitized region were gathered in a single x-ray spectrum. Matrix corrections, and application of standards used ThermoFisher software. Finally, WDS mapping results were collected with the ProbeImage software, and the data were processed using ProbeforEPMA, and CalcImage software. WDS mapping data were acquired using $15 \mathrm{kV}$ energy, with $100 \mathrm{nA}$ current, with Scott-Love matrix corrections. Matrix corrections are applied to each pixel individually.

Results obtained by each method are shown in Table 1 . These results generally show reasonable agreement between the various methods for most elements. It should be noted that we don't have independent results for the "true" bulk composition of any of these objects, so that it is impossible to firmly assess which method produces the closest approach to the true answer. Here, we show that these methods produce results that are quite similar, and note and assess the sources of potential error imbedded in each method. In the end, it is suspected that the best method is by quantification of WDS-mapping data (despite lower totals). Matrix corrections are applied in this method for each pixel independently, unlike the other two methods, where 
corrections are applied to mixed-phase analyses. Issues that are particular to the EDS mapping method are noted below. The background correction applied to the EDS x-ray signals are modelled by the ThermoFisher software, and we have experience showing that this background model can be problematic. This issue can produce particularly bad results for minor elements, such as sulfur, potassium and phosphorus in the examples shown below (bold numbers in the table). Additionally, the ED detector, at high count rates, produces sum and escape peaks. The NSS software removes sum and escape peaks, but this process can be imperfect. For instance, the $\mathrm{O}+\mathrm{O}$ sum peak resides at the same position as the $\mathrm{Na} \mathrm{K} \alpha$ peak, and the $\mathrm{Al}+\mathrm{O}$ sum peak is at the same energy as the $\mathrm{P} \mathrm{K} \alpha$ peak. Similarly, the $\mathrm{Si}+\mathrm{Al}$ sum peak overlaps the position of the potassium peak, and if the sum peak removal is flawed this can result in errors for potassium abundance.

\section{References:}

[1] P Duncumb and SJB Reed, Quantitative Electron Probe Microanalysis, NBS Special Pub. 298 (1968) ed. KFJ Heinrich, p. 133.

[2] JL Pouchou and F. Pichoir, Scanning 12 (1990), p. 212.

[3] GF Bastin and HJM Heijligers, Electron Probe Quantitation (1991) eds. KFJ Heinrich and DE Newbury, p.145.

Table 1: Comparison of various methods for determining bulk composition

\begin{tabular}{lrccrcccccc}
\hline & \multicolumn{3}{c}{ ARC1 } & \multicolumn{3}{c}{ ARC2 } & \multicolumn{3}{c}{ ARC3 } \\
\hline & defocus & ED map & WD map & defocus & ED map & WD map & defocus & ED map & WD map \\
$\mathrm{SiO}_{2}$ & 40.48 & 42.32 & 40.49 & 41.50 & 43.62 & 41.92 & 44.46 & 44.85 & 43.44 \\
$\mathrm{TiO}_{2}$ & 0.50 & 0.50 & 0.47 & 0.65 & 0.77 & 0.71 & 0.95 & 0.72 & 0.91 \\
$\mathrm{P}_{2} \mathrm{O}_{5}$ & 0.03 & $\mathbf{0 . 2 7}$ & nd & 0.01 & $\mathbf{0 . 4 3}$ & nd & 0.01 & $\mathbf{0 . 1 2}$ & nd \\
$\mathrm{Al}_{2} \mathrm{O}_{3}$ & 13.11 & 12.48 & 10.79 & 19.55 & 18.45 & 17.73 & 17.47 & 18.66 & 17.51 \\
$\mathrm{Cr}_{2} \mathrm{O}_{3}$ & 0.16 & $\mathbf{0 . 0 4}$ & 0.18 & 0.13 & 0.15 & 0.14 & 0.19 & $\mathbf{0 . 0 3}$ & 0.18 \\
$\mathrm{FeO}$ & 0.90 & 1.67 & 3.02 & 2.46 & 3.13 & 3.86 & 4.18 & 4.40 & 5.06 \\
$\mathrm{MnO}$ & 0.02 & bdl & 0.03 & 0.02 & 0.04 & 0.03 & 0.05 & bdl & 0.06 \\
$\mathrm{MgO}$ & 34.45 & 34.26 & 32.42 & 22.73 & 20.21 & 20.35 & 15.32 & 14.92 & 14.62 \\
$\mathrm{CaO}$ & 8.35 & 8.87 & 8.72 & 12.22 & 13.47 & 12.81 & 15.45 & 14.46 & 14.05 \\
$\mathrm{Na} 2 \mathrm{O}$ & 1.15 & $\mathbf{0 . 4 3}$ & 1.24 & 0.43 & 0.70 & 0.45 & 2.17 & 2.62 & 2.39 \\
$\mathrm{~K} 2$ & 0.05 & 0.02 & nd & 0.02 & $\mathbf{0 . 2 0}$ & nd & 0.06 & 0.05 & nd \\
$\mathrm{S}$ & 0.04 & $\mathbf{0 . 2 6}$ & nd & 0.03 & $\mathbf{0 . 2 8}$ & nd & 0.07 & bdl & nd \\
$\mathrm{Cl}$ & nd & bdl & 0.01 & nd & $\mathbf{0 . 1 5}$ & 0.04 & nd & 0.02 & 0.07 \\
$\mathrm{Total}$ & 99.24 & 101.12 & 97.36 & 99.75 & 101.60 & 98.04 & 100.38 & 100.55 & 98.59 \\
\hline
\end{tabular}

Notes: nd indicates not determined, and bdl indicates below detection limits. Examples of particularly bad disagreement are highlighted in bold.
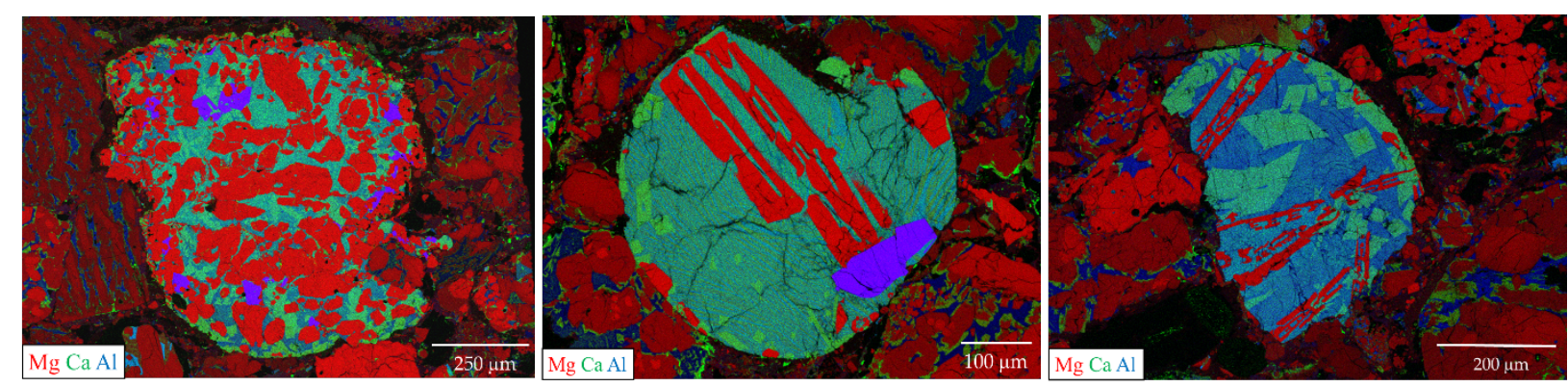

Figure 1. EDS-derived x-ray maps of 3 Al-rich chondrules in the unequilibrated ordinary chondrite NWA7402. Forsterite $\left(\mathrm{Mg}_{2} \mathrm{SiO}_{4}\right)$ is bright-red, spinel $\left(\mathrm{MgAl}_{2} \mathrm{O} 4\right)$ is purple, clinopyroxene $\left(\mathrm{CaMgSi}_{2} \mathrm{O}_{6}\right)$ is bright green, and the partly glassy, quench-crystallized matrix of each chondrule is green-blue to blueish. $\mathrm{Mg}$ is in red, $\mathrm{Ca}$ in green and $\mathrm{Al}$ in blue in these tri-color maps. 